\title{
Development of a solid-phase microextraction fiber by chemical binding of polymeric ionic liquid on a silica coated stainless steel wire
}

\author{
Long Pang, Jing-Fu Liu*

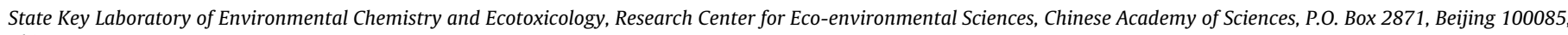
China

\section{A R T I C L E I N F O}

\section{Article history:}

Received 28 November 2011

Received in revised form 17 January 2012

Accepted 19 January 2012

Available online 25 January 2012

\section{Keywords:}

Polymeric ionic liquid

Solid-phase microextraction

Polycyclic aromatic hydrocarbons

Fiber coating

\begin{abstract}
A B S T R A C T
A novel approach was developed for the fabrication of solid-phase microextraction (SPME) fiber by coating stainless steel fiber with a polymeric ionic liquid (PIL) through covalent bond. The stainless steel fiber was sequentially coated with a gold film by replacement reaction between Fe and Au when immerged in chloroauric acid, assembled with a monolayer of 3-(mercaptopropyl) triethoxysilane on the gold layer through the Au-S bond, and coated with a silica layer by the hydrolysis and polycondensation reaction of the surface-bonded siloxane moieties and the active silicate solution. Then, 1-vinyl-3-(3-triethoxysilylpropyl)-4,5-dihydroimidazolium chloride ionic liquid was anchored on the silica layer by covalent bond, and the PIL film was further formed by free radical copolymerization between 1-vinyl-3-(3-triethoxysilylpropyl)-4,5-dihydroimidazdium and vinyl-substituted imidazolium with azobisisobutyronitrile (AIBN) as initiator. Parameters influencing the preparation of PIL fiber were optimized, and the developed SPME fiber has a coating thickness of $\sim 20 \mu \mathrm{m}$ with good thermal stability and long lifetime. The performance of the PIL fiber was evaluated by analysis of polycyclic aromatic hydrocarbons (PAHs) in water samples. The developed PIL fiber showed good linearity between 0.5 and $20 \mu \mathrm{g} \mathrm{l}^{-1}$ with regression coefficient in the range of $0.963-0.999$, detection limit ranging from 0.05 to $0.25 \mu \mathrm{g} \mathrm{I}^{-1}$, and relative standard deviation of $9.2-29 \%(n=7)$. This developed PIL fiber exhibited comparable analytical performance to commercial $7 \mu \mathrm{m}$ thickness PDMS fiber in the extraction of PAHs. The spiked recoveries for three real water samples at $0.5-5 \mu \mathrm{gl}^{-1}$ levels were $49.6-111 \%$ for the PIL fiber and $40.8-103 \%$ for the commercial PDMS fiber.
\end{abstract}

(c) 2012 Elsevier B.V. All rights reserved.

\section{Introduction}

Ionic liquids (ILs) are compounds composed of organic cations and organic or inorganic anions that have melting points below $100^{\circ} \mathrm{C}$. ILs have negligible vapor pressures at room temperature and high thermal stability, and are considered as "green solvents" in comparison with volatile organic solvents [1]. Owing to these unique properties, ILs have been applied in organic synthesis [2], separation [3], electrochemistry [4], and catalysis [5]. ILs also exhibit good performance in the field of analytical chemistry [6-9], including sample preparation [10-13], chromatographic/capillary electrophoretic separation [14-16] and detection [17].

Many researches have illustrated that ILs are promising solvents in sample preparation including liquid-liquid extraction (LLE) $[18,19]$, liquid phase microextraction (LPME) [20-22], and solid phase microextraction (SPME) [23-26]. Liu et al. [23] for the first time reported headspace SPME with IL-coated fiber. Briefly, a

\footnotetext{
* Corresponding author. Tel.: +86 10 62849192; fax: +861062849192.

E-mail address: jfliu@rcees.ac.cn (J.-F. Liu).
}

stainless steel wire was coated with ILs layer prior to extraction, and then the fiber was desorbed on the injection port of GC. The performance of this proposed fiber is comparable with that of commercial PDMS fibers, but the coating is disposable and has to be prepared for each extraction. In addition, the physical adsorption-based coating gives rise to loss of ILs on the desorbed process in the injection port of GC. In order to overcome these disadvantages, Kuei et al. [24] utilized Nafion membrane as supporter to enhance the amount and stability of ionic liquid film on the fiber. Though the presence of Nafion membrane enhanced the extracted amount of target compounds with factors about $2-3$, repeated coating and washing process had to be conducted for each extraction. To overcome this problem, polymeric ionic liquids (PILs) were introduced as novel coating materials for SPME by Anderson and coworkers [25,26]. Compared to IL-based SPME fiber, the PIL-based SPME fiber exhibited exceptional stability and long lifetimes, avoiding the leaching of coating materials into the injection port and the contamination of the GC column.

Currently, most IL-based SPME fibers were prepared by physical adsorption-based coating which has limited long-term stability. This limitation can be overcome by immobilizing the ILs on the 
fiber by the chemical bond, which has been proved to efficiently reduce the leaching of incorporated ILs from catalyst [27-32] As examples, alkoxysilyl-functionalized 4,5-dehydroimidazolium salts were grafted onto the silica gel by covalent bonds between silanol groups and the cations of the "task-specific" ILs, and the rhodium-catalyzed supported IL materials were applied in catalysis [29]. In addition, the chloroaluminate ILs were immobilized on inorganic supports $\left(\mathrm{SiO}_{2}, \mathrm{Al}_{2} \mathrm{O}_{3}, \mathrm{TiO}_{2}, \mathrm{ZrO}_{2}\right)$ using triethoxysilylfunctionalized imidazolium salts [30].

In this present study, we developed a novel approach for constructing the PIL-based SPME fiber through chemical binding. The stainless steel fiber was coated by following steps in sequence: (i) coating with gold by replacement reaction in chloroauric acid; (ii) coating with 3-(mercaptopropyl) triethoxysilane through the Au-S bond; (iii) formation of $\mathrm{SiO}_{2}$ film by $\mathrm{Si}-\mathrm{O}-\mathrm{Si}$ bond; (iv) anchoring 1vinyl-3-(3-triethoxysilylpropyl)-4,5-dihydroimidazdium chloride IL on the silica layer by covalent bond; and (v) formation of PIL film by free radical copolymerization between 1-vinyl-3- (3-triethoxysilylpropyl)-4,5-dihydroimidazdium and vinyl-substituted imidazolium with azobisisobutyronitrile (AIBN) as initiator, which enhance the amount of polymeric ionic liquids (PIL) on the fiber [33]. The performance of the home-made PIL-coated SPME fiber was evaluated by comparison with the commercial PDMS fiber in the extraction of PAHs in aqueous samples.

\section{Experimental}

\subsection{Reagents}

The standard mixtures of 16 PAHs at a concentration of $0.2 \mathrm{mg} \mathrm{ml}^{-1}$ in methylene chloride/methanol $(1 / 1, \mathrm{v} / \mathrm{v})$ were purchased from Accus Standard (New Haven, USA). 1-Vinylimidazole was obtained from J \& K Chemical (Logan, Utah, USA). 3Chloropropyltriethoxysilane and 3-(mercaptopropyl) triethoxysilane were purchased from TCI Development Co., Ltd. (Tokyo, Japan). Lithium bis (trifluoromethanesolphonyl) imide ( $\left.\operatorname{LiNTf}_{2}\right)$ was obtained from Shanghai Cheng Jie Chemical Co. Ltd (Shanghai, China). All other reagents including azobisisobutyronitrile, 1-chlorobutane, sodium silicate, hydrochloric acid and sodium hydroxide were all purchased from Sinopharm Chemical Reagent Co., Ltd. (Beijing, China). The chemicals were of analytical grade or above unless otherwise stated. Deionized water was obtained from a Milli-Q water purification system (Millipore, Bedford, MA, USA).

\subsection{Preparation of the SPME fiber}

\subsubsection{Coating a silica film on stainless steel fiber}

The coating of silica film on stainless steel fiber was carried out by using a modified procedures described elsewhere [34]. Briefly, a stainless steel wire $(0.15 \mathrm{~mm}$ ID) was polished smoothly and washed with methanol and dichloromethane successively prior to coating, and then conditioned under vacuum at $200^{\circ} \mathrm{C}$. After removing from vacuum oven and cooled to room temperature, the wire was dipped into chloroauric acid ( $1 \mathrm{mM}$, anhydrous ethanol as solvent) and held for $2 \mathrm{~h}$. Then the fiber was immerged into a freshly prepared 3-mercaptopropyltriethoxysilane solution (1 $\mathrm{mM}$, in anhydrous ethanol) and shaken for $60 \mathrm{~min}$. Afterwards, the wire was transferred into the active silicate solution, which was obtained by tuning the sodium silicate solution $(100 \mathrm{mM})$ to $\mathrm{pH} 9$ by progressive addition of $1 \mathrm{M} \mathrm{HCl}$. After shaking for at least $24 \mathrm{~h}$, the silica layer was polymerized on the gold layer gradually, as sketched in the Fig. S1.

\subsubsection{Synthesis of IL monomers and polymers}

IL monomer, 1-vinyl-3-butylimidazdium chloride was synthesized as described in literature [35]. In briefly, 1-vinylimidazole $(0.05 \mathrm{~mol})$ and 1 -chlorobutane $(0.10 \mathrm{~mol})$ were mixed in $20 \mathrm{ml}$ 2-propanol and transferred to a flask with round bottom and stirred vigorously for $50 \mathrm{~h}$ at $70^{\circ} \mathrm{C}$ under nitrogen atmosphere. After cooling to room temperature, phase separation occurred and the product was obtained as a viscous liquid, which was filtered and dried in a vacuum oven until constant weight. 1-Vinyl3-butylimidazdium chloride was obtained as a white solid. For polymerization of ILs monomers, the IL monomers $(3.0 \mathrm{~g})$ were mixed with chloroform $(30 \mathrm{ml})$, and then into the mixture was added the AIBN initiator at $70^{\circ} \mathrm{C}$ under nitrogen atmosphere. After vigorously stirring for $3 \mathrm{~h}$, the mixture was evaporated under vacuum and the residual was dried in a vacuum oven at $60^{\circ} \mathrm{C}$ (Fig. 1a). As shown in Fig. 1b, the halide anion was exchanged with bis [(trifluoromethy) sulfonyl] imide anion [ $\left.\mathrm{NTf}_{2}{ }^{-}\right]$ by metathesis anion exchange. In this experiment, the 1-vinyl-3butylimidazdium chloride was dissolved in acetonitrile and treated with one equivalent of lithium bis (trifluoromethanesolphonyl) imide. The mixture solution was stirred overnight at room temperature and the final product was obtained by filtration process and reduced pressure distillation. 1-Vinyl-3-(3-triethoxysilylpropyl)4,5-dihydroimidazolium chloride was synthesized with the similar procedure as the literature reported by Shen [36]. Briefly, 1vinylimidazole $(9.4 \mathrm{~g}, 0.1 \mathrm{~mol})$ and chloropropyltriethoxysilane $(24.1 \mathrm{~g}, 0.1 \mathrm{~mol})$ were placed in a $50 \mathrm{ml}$ stainless steel autoclave, which was sealed and heated to $120^{\circ} \mathrm{C}$ for $24 \mathrm{~h}$. The sticky residue was washed with ethyl acetate and diethyl ether for several times. Extraction of the material into dichloromethane $(100 \mathrm{ml})$ and filtration through a bed of activated carbon and alumina gave a brown colored paste after the removal of the volatile components under reduced pressure. Finally, the product was dried at $60^{\circ} \mathrm{C}$ in vacuum oven until constant weight (Fig. $1 \mathrm{c}$ ).

a

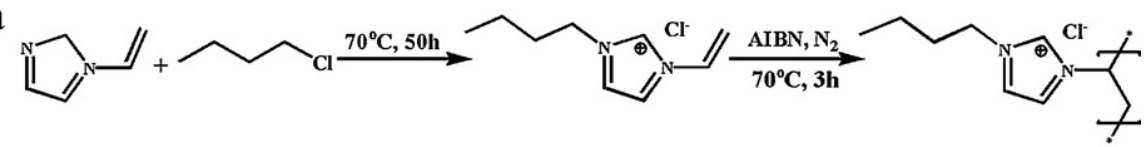

b

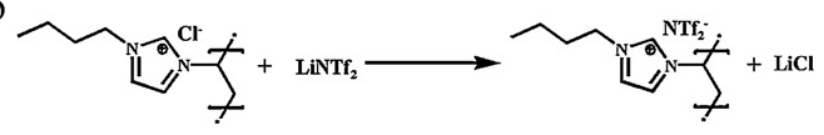

C

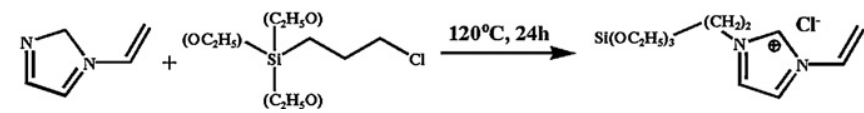

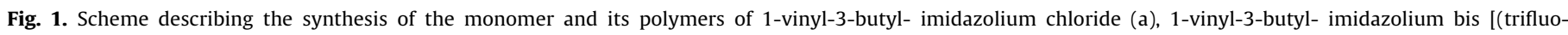
romethyl)sulfonyl] imide ( $\mathrm{NTf}_{2}^{-}$) (b), and 1-vinyl-3-(3-triethoxysilylpropyl)-4,5-dihydroimidazdium chloride (c). 

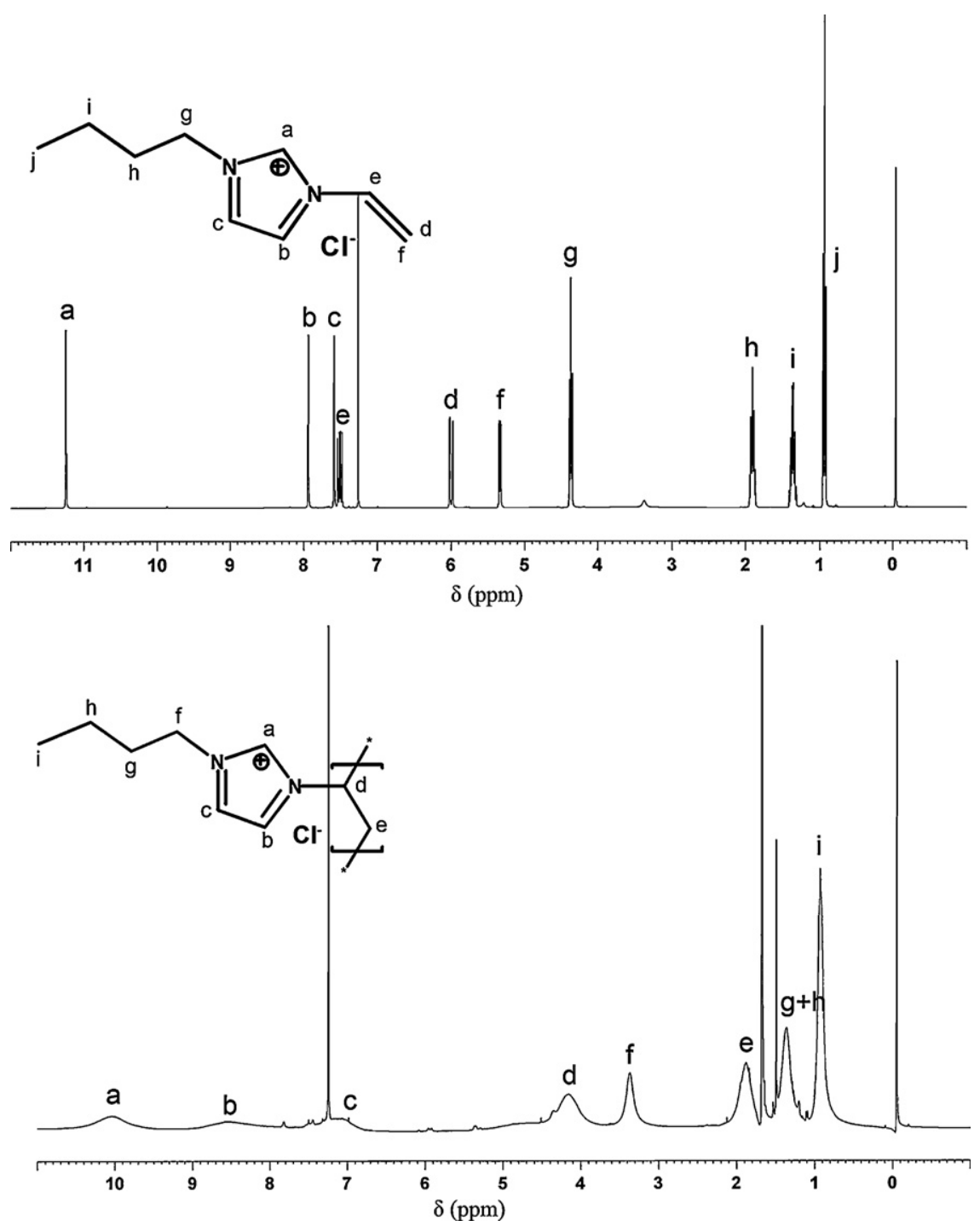

Fig. 2. ${ }^{1} \mathrm{H}$ NMR spectra (400-MHz) of the syhthesized 1-vinyl-3-butyl-imidazolium chloride and poly (1-vinyl-3-butyl- imidazolium) chloride.

The ${ }^{1} \mathrm{H}$ NMR spectra of the 1-vinyl-3-butylimidazdium chloride monomer and the corresponding poly (1-vinyl-3butylimidazdium) chloride polymer were shown in Fig. 2. The disappearance of the double bond originating from the vinylsubstituted monomer and the broadening of the signals illustrated the formation of polymer. The monomer and polymer were characterized using ${ }^{1} \mathrm{H}$ NMR [ $\delta \mathrm{ppm}$ relative to TMS]: 1-vinyl3-butylimidazdium chloride (400 MHz, d6-CDCl $): 11.241(\mathrm{~s}, 1 \mathrm{H})$, 7.937(s, 1H), 7.586(s, 1H), 7.500(dd, 1H), 6.018(dd, 1H), 5.347(dd, $1 \mathrm{H}), 4.370(\mathrm{t}, 1 \mathrm{H}), 1.910(\mathrm{~m}, 2 \mathrm{H}), 1.357(\mathrm{~m}, 2 \mathrm{H}), 0.935(\mathrm{t}, 3 \mathrm{H})$.

\subsubsection{Preparation of PIL-coated fibers}

The PIL-coated fiber was prepared by two main steps (Fig. S2), i.e. the immobilization of 1-vinyl-3-(3-triethoxysilylpropyl)-4,5dihydroimidazdium chloride and the polymerization 1-vinyl-3butylimidazdium chloride. Firstly, the above prepared stainless steel fiber with silica shell was completely immerged into 1vinyl-3-(3-triethoxysilylpropyl)-4,5-dihydroimidazolium chloride solution ( $10 \mathrm{mmol}$, the chloroform), and the mixtures were refluxed for $16 \mathrm{~h}$ under reflux condition. By this procedure, the 1-vinyl3-(3-triethoxysilylpropyl)-4,5-dihydroimidazolium chloride was "grafted" on the surface of the fiber by covalent bond. Then, the 1vinyl-3-(3-triethoxysilylpropyl)-4,5-dihydroimidazolium chloride on the fiber was copolymerized with 1-vinyl-3-butylimidazdium chloride by free radical reaction in the presence of AIBN at $70^{\circ} \mathrm{C}$ for $3 \mathrm{~h}$ under nitrogen atmosphere. After that, the fiber with polymer coatings was removed from the mixture and held in air for $10 \mathrm{~min}$ to evaporate residual chloroform. The halide anion of copolymer was exchanged with bis[(trifluoromethyl)sulfonyl]imide $\left(\mathrm{NTf}_{2}{ }^{-}\right)$ anion by overnight stirring in equimolar amount of lithium bis[(trifluoromethyl)sulfonyl]imide ( LiNTf $_{2}$ ) solution. Finally, the fiber was conditioned at $280^{\circ} \mathrm{C}$ under nitrogen for $10 \mathrm{~min}$ in the gas chromatograph (GC) injection port in order to remove the residual volatile solvents.

\subsection{Characterization of the fiber}

The surface morphology and elemental analysis of the fiber were accomplished by using a Hitachi S-3000N equipped with energy dispersive spectrometer (EDS) (Hitachi, Japan). A confocal Raman spectroscopy system (Renishaw InVia Raman microscope, UK) was utilized to prove the coating procedure of silica layer. The characterization of ionic liquids and polymers were conducted by a Bruker UltraShield 400 plus (400-MHz) spectrometer (Bruker, Germany). 


\subsection{Solid-phase microextraction procedures}

To perform the SPME of PAHs, into a $40 \mathrm{ml}$ extraction vial was placed $35 \mathrm{ml}$ water sample or standards solutions which contained $5 \mu \mathrm{g} \mathrm{l}^{-1}$ each of the $16 \mathrm{PAHs}$, and $1.5 \mathrm{~cm}$ long PTFE coated stir bar. The vial was rapidly sealed with a cap wrapped with aluminum foil and then put on the magnetic stirrer for stirring at $1000 \mathrm{rpm}$. The septum piercing needle of the SPME device was introduced into the vial and the fiber was completely immersed into the aqueous phase. After extraction for $50 \mathrm{~min}$ at $20^{\circ} \mathrm{C}$, the fiber was transferred to the GC port for analytes desorption and further analysis. Each extraction and determination was repeated three times.

\subsection{GC-MS analysis of PAHS}

Quantification of PAHs compounds was performance by an Agilent 7890A GC coupled to an Agilent 5975C mass spectrometer (MS) detector, which equipped with a HP-5MS column $(30 \mathrm{~m} \times 0.25 \mathrm{~mm}$, $0.25 \mu \mathrm{m}$ film thickness). The oven temperature was initially held at $50{ }^{\circ} \mathrm{C}$ for $2 \mathrm{~min}$, increased to $200^{\circ} \mathrm{C}$ at a rate of $19^{\circ} \mathrm{C} \mathrm{min}^{-1}$, ramped at $4.5^{\circ} \mathrm{C} \mathrm{min}^{-1}$ to $240^{\circ} \mathrm{C}$ and held for $2 \mathrm{~min}$, and finally ramped to $290^{\circ} \mathrm{C}$ at a rate of $2.5^{\circ} \mathrm{C} \mathrm{min}^{-1}$ and held for $2 \mathrm{~min}$. The ionization was carried out in the electron impact mode $(70 \mathrm{eV})$. The temperatures of injector, transfer lines and ion-source were set at $280^{\circ} \mathrm{C}$, $280^{\circ} \mathrm{C}$ and $230^{\circ} \mathrm{C}$, respectively. Helium was used as carried gas and at flow rate of $0.79 \mathrm{ml} \mathrm{min}^{-1}$. The GC was operated in the splitless mode and the splitless time was $1 \mathrm{~min}$. The selected-ion monitoring (SIM) mode was carried out for the quantification of PAHs in environmental water samples. The retention time and specific ion $m / z$ ratio of each compound were shown in Table 1 .

\subsection{Sample collection}

Three environmental water samples were collected, including river water, ground water, and municipal sewage water samples. River water sample was collected from Hulanhe River, a branch of Songhuajiang River located in the northeast of China. The effluent of municipal sewage water sample was collected from Qinghe sewage treatment plant located in the northwest of Beijing. Ground water sample was obtained from a site of coke oven plant located in the southeast of Beijing.

Table 1

Quantitation ion and retention time for the GC-MS determination of the studied PAHs.

\begin{tabular}{|c|c|c|c|}
\hline Compounds & Abbreviation & $\begin{array}{l}\text { Retention } \\
\text { time (min) }\end{array}$ & $\begin{array}{l}\text { Quantization ion }(\mathrm{m} / \mathrm{z}) \text { for } \\
\text { full and SIM mode }\end{array}$ \\
\hline Naphthalene & Nap & 7.61 & 128 \\
\hline Acenaphthylene & Acpy & 9.84 & 152 \\
\hline Acenaphthene & Acp & 10.11 & 153 \\
\hline Fluorine & Flu & 10.92 & 166 \\
\hline Anthracene & Ant & 12.98 & 178 \\
\hline Phenanthrene & $\mathrm{Pa}$ & 13.10 & 178 \\
\hline Fluoranthene & $\mathrm{Fl}$ & 16.87 & 202 \\
\hline Pyrene & Pyr & 17.72 & 202 \\
\hline $\begin{array}{l}\text { Benz }[a] \\
\text { anthracene }\end{array}$ & Baa & 23.56 & 228 \\
\hline Chrysene & Chr & 23.80 & 228 \\
\hline $\begin{array}{l}\text { Benzo }[b] \\
\text { fluoranthene }\end{array}$ & Bbf & 30.62 & 252 \\
\hline $\begin{array}{l}\text { Benzo }[k] \\
\text { fluoranthene }\end{array}$ & Bkf & 30.80 & 252 \\
\hline Benzo $[a]$ pyrene & Bap & 32.69 & 252 \\
\hline $\begin{array}{l}\text { Benzo [ghi] } \\
\text { perylene }\end{array}$ & Bjhip & 40.18 & 276 \\
\hline $\begin{array}{l}\text { Dibenzo }[a, h] \\
\text { anthracene }\end{array}$ & Dba & 40.55 & 278 \\
\hline $\begin{array}{l}\text { Indeno }[1,2,3-c d] \\
\text { pyrene }\end{array}$ & Ind & 41.69 & 276 \\
\hline
\end{tabular}

\section{Results and discussion}

\subsection{Coating a silica film on the surface of stainless wire}

Prior to coat a smooth film of silica layer, the surface of stainless steel fiber was coated with gold and 3-(mercaptopropyl) triethoxysilane in sequence. Some parameters influencing the coating processes such as the replacement reaction time in chloroauric acid, the concentration and $\mathrm{pH}$ of the sodium silicate, and the coating time of silica layer were studied. The effects of parameters on the coated silica film were evaluated by the coated silica amount that was tested by EDS and Raman spectra.

For coating with a gold layer, the stainless steel wire was immerged in a chloroauric acid solution $(1 \mathrm{mM}$, in anhydrous ethanol). The replacement reaction time was optimized in the range of 1-8 h, and SEM-EDS results showed that the reaction occurred immediately and a smooth gold layer was formed in $2 \mathrm{~h}$. In order to save time, $2 \mathrm{~h}$ was selected.

The gold coated stainless fiber was further immersed into the silane coupling agent 3-mercaptopropyltriethoxysilane $(1 \mathrm{mM}$, in anhydrous ethanol) and kept shaking for $60 \mathrm{~min}$, instead of 15 min adopted in the literature reported [34]. This prolonged time ensured the binding of 3-mercaptopropyltriethoxysilane on the gold layer through complexation of the mercapto group with gold. No further experiments were carried out for the optimal condition in this work. The coated 3-mercaptopropyl-triethoxysilane functionalized as a primer for the coating of silica film on the gold layer.

The sodium silicate concentration and $\mathrm{pH}$ strongly affect the formation of active silica (silicate groups for polymerization), which formed by decreasing the $\mathrm{pH}$ of sodium silicate solution by progressive addition of $1 \mathrm{M} \mathrm{HCl}$. The active silica polymerized immediately with silane triols formed by the hydrolysis of the surface-bonded siloxane moieties and thus form a silica layer on the gold layer of the stainless fiber. The concentration of sodium silicate was optimized in the range of $1-200 \mathrm{mM}$ at $\mathrm{pH} \mathrm{9,} \mathrm{and} \mathrm{results} \mathrm{shown} \mathrm{in}$ Fig. S3 (a) indicated that the coated silica increased significantly with the increase of sodium silicate concentration from $50 \mathrm{mM}$ to $100 \mathrm{mM}$. Further increase of sodium silicate concentration to $200 \mathrm{mM}$, however, gave rise to the formation of silica gel and thus worse repeatability due to the polymerization of silicate groups. Therefore, $100 \mathrm{mM}$ of sodium silicate was utilized in the following work. The $\mathrm{pH}$ of sodium silicate was optimized in the range of 8.0-12.8. As can be seen from Fig. S3 (b), the coated amount of silica decreased with the increase of $\mathrm{pH}$. Although the coated silica amount at $\mathrm{pH} 9.0$ was lower than that at $\mathrm{pH} 8.0, \mathrm{pH} 9.0$ provided much better repeatability than $\mathrm{pH} 8.0$ with the similar reason of sodium silicate at high concentration that silica gel was formed at $\mathrm{pH}$ 8.0. Thus, pH 9.0 was adopted in the following studies.

Given the formation of silica layer is a time-consuming process, the coating time of silica layer was optimized in this study. As can be seen from the Fig. S3 (c), variation of coating time in the range of 1-9 days has no significant influence on the silica percentage of the fiber surface. Accordingly, a coating time of $1 \mathrm{~d}$ was utilized in the following studies.

Fig. 3 shows the SEM image of the formed silica layer that was rough and porous, resulting from the oligomeric nature of active silica and the occurrence of direct adsorption of silica oligomers during the aging process. SEM test also showed that the coating thickness of this rough and porous silica was approximately $3 \mu \mathrm{m}$, and EDS test showed that with $\sim 1.5 \mathrm{wt} \% \mathrm{Si}$. Raman spectra was also presented to prove the coating procedure of silica layer (Fig. S4). In the Raman spectra of (3-mercaptopropyl) triethoxysilane, the peak in the range of $2600-2500 \mathrm{~cm}^{-1}$ was assigned to the $\mathrm{S}-\mathrm{H}$ bond, while the peaks in the range of $3000-2800 \mathrm{~cm}^{-1}$ were assigned to $-\mathrm{CH}_{3}$ and $-\mathrm{CH}_{2}$. Fig. $\mathrm{S} 4$ (B) shows the Raman spectra of the stainless 

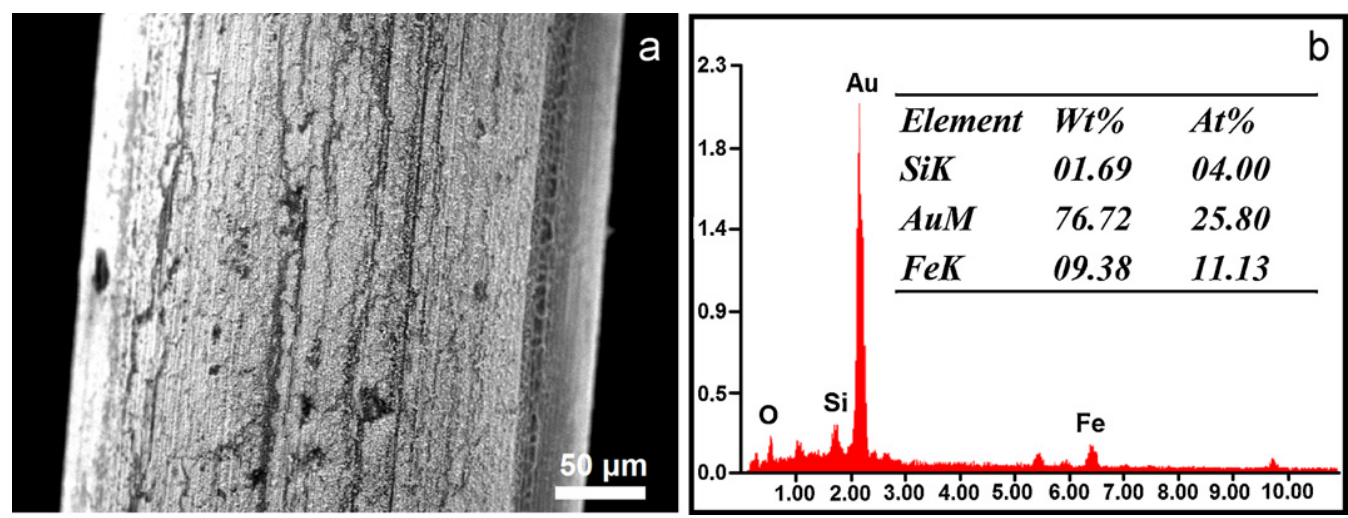

Fig. 3. Scanning electron microscopy images and EDS test results of the stainless steel wire coated with a silica layer under optimal conditions.

steel fiber that was covered with gold film and silica layer (record a) and a stainless steel fiber used as control (record b). The presence of characteristic peak at $1100-1000 \mathrm{~cm}^{-1}$ demonstrated the presence of $\mathrm{Si}-\mathrm{O}$ bond, while the absence of characteristic peak at $2600-2500 \mathrm{~cm}^{-1}$ suggests that the $\mathrm{S}-\mathrm{H}$ bond disappeared due to the formation of Au-S bond. Accordingly, it can be concluded that the underling reactions involved in the coating procedure of silica layer.

\subsection{Preparation of the PIL coatings}

Anchoring 1-vinyl-3-(3-triethoxysilylpropyl)-4,5-dihydroimidazdium chloride on the silica layer by covalent bond was based on the literature method [30], no further optimization was carried out in this work. As the fiber immobilized with only a monolayer of 1-vinyl-3-(3-triethoxysilylpropyl)-4,5dihydroimidazdium chloride showed low extraction efficiencies toward analytes due to the relatively thin coating film, the 1vinyl-3- (3-triethoxysilylpropyl)-4,5-dihydroimidazdium chloride coated on the fiber was copolymerized with 1-vinyl-3butylimidazdium chloride for 0,1 , and $3 \mathrm{~h}$, respectively to improve thicker PIL film and thus the extraction efficiency.

Fig. 4 shows the SEM images of the fiber at different stages. The PIL film is a porous layer whose film thickness increased with the polymerization time. SEM tests showed that the thickness of the PIL coatings obtained with 1 and $3 \mathrm{~h}$ copolymerization were approximately 7 and $20 \mu \mathrm{m}$, respectively. The performance of the obtained PIL-coated SPME fibers were tested by extraction of PAHs. Results showed in Fig. 5 indicate that for the low-ring PAHs, the signal of extracted PAHs increased with copolymerization time. This is
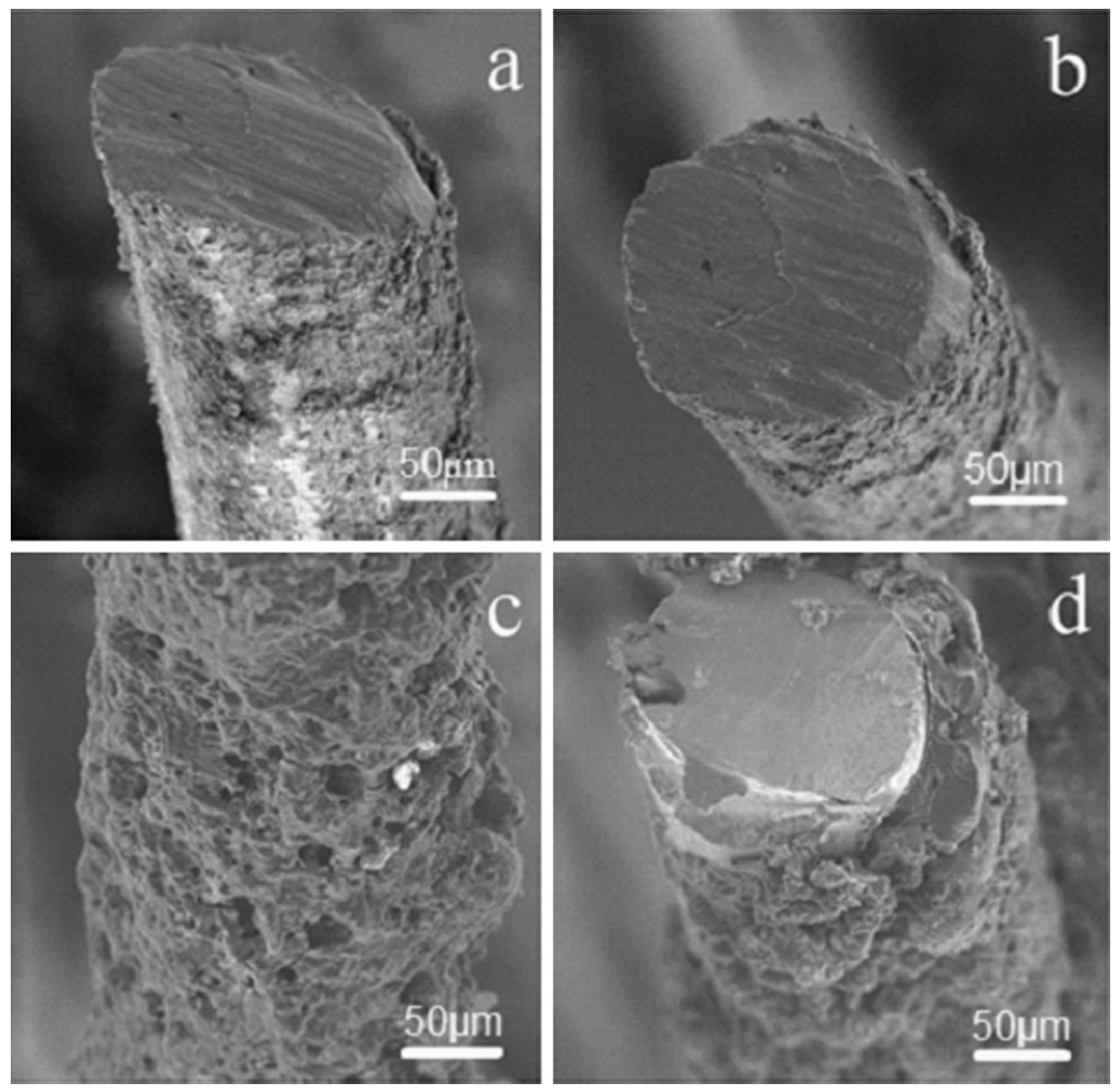

Fig. 4. SEM images of the SPME fibers prepared by copolymerized with PIL for $1 \mathrm{~h}(\mathrm{a}, \mathrm{b})$, and $3 \mathrm{~h}(\mathrm{c}, \mathrm{d})$, respectively. 


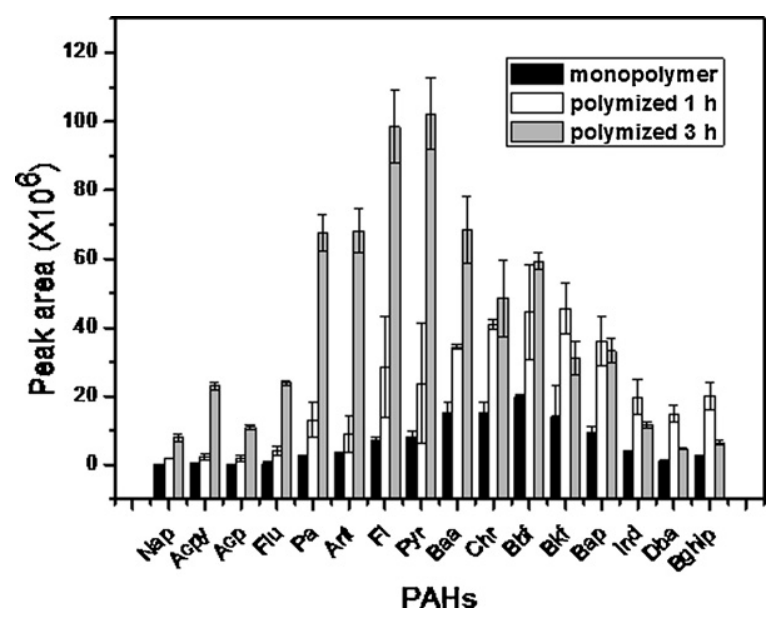

Fig. 5. Extraction performance for PAHs with the home-made SPME fibers prepared by copolymerized with PIL for $0 \mathrm{~h}$ (monopolymer), $1 \mathrm{~h}$, and $3 \mathrm{~h}$, respectively.

because the thickness of PIL coating increased with copolymerization time, and the extracted amount of low-ring PAHs increased with the PIL coating thickness as they have relatively high diffusion rates. For the high-ring PAHs, possessing of strong hydrophobic, relative thin PIL film was prone to make them reach balance between fiber and aquatic solution. Whereas, compared to low-ring PAHs, there was no remarkable improvement on high-ring PAHs between polymerization $1 \mathrm{~h}$ and $3 \mathrm{~h}$. Thus, in the following studies, at copolymerization time of $3 \mathrm{~h}$ was adopted.

\subsection{Analytical performance}

Table 2 illustrated the parameters of the calibration curves for PAH analysis with the home-made PIL-coated fiber. The linearity of the method was tested by GC-MS using standard solution with concentrations in the range of $0.5-20 \mu \mathrm{g} \mathrm{l}^{-1}$ for low-ring PAHs and $0.5-10 \mu \mathrm{gl}^{-1}$ for high-ring PAHs, respectively. The proposed method showed good linearity with regression coefficients ranging between 0.966 and 0.999 . Owing to the low water solubility and long equilibration time of the high-ring PAHs, their correlation coefficients were relatively low. The limits of detection (LODs) were in the range of $0.05-0.25 \mu \mathrm{gl}^{-1}(\mathrm{~S} / \mathrm{N}=3)$. The precision of the method, evaluated by the relative standard deviations (RSDs) determined by performing seven consecutive extractions of standard solutions spiked with $5 \mu \mathrm{g} \mathrm{l}^{-1}$ each of the tested PAHs, were in the

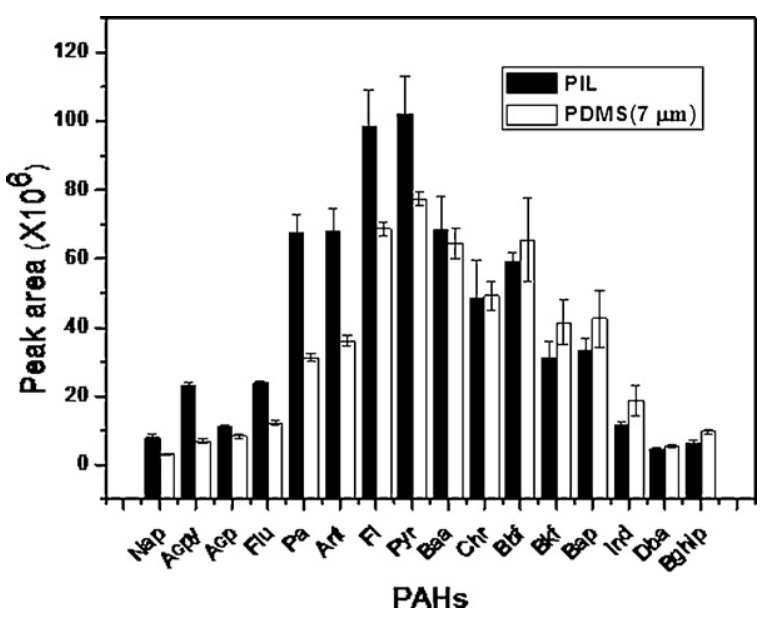

Fig. 6. Comparison of the extraction performance for PAHs with the home-made PIL fiber (copolymerized $3 \mathrm{~h}$ ) and commercial fiber ( $7 \mu \mathrm{m}$ PDMS).

range of 9-29\%. The fiber-to-fiber reproducibility of the PIL-coated fiber, tested by determining the RSDs of four fibers prepared in the same batch in analysis of $5 \mu \mathrm{g} \mathrm{l}^{-1}$ PAHs, were in the range of 9-46\% (Table S1), and the fiber-to-fiber reproducibility can be improved by further optimization of the preparation procedures. The homemade fiber have comparable lifetime with the commercial fibers (Fig. S5). After 40 times extraction, the signals (peak area) reduced $20-58 \%$ for the home-made fiber and $23-52 \%$ for the commercial PDMS fiber, respectively.

The analytical performance of this home-made PIL-based fiber was compared with the $7-\mu \mathrm{m}$ thickness commercial PDMS fiber. Fig. 6 shows that the PIL fiber exhibited greater extraction ability for low-ring PAHs. In comparison to the commercial PDMS fiber, the PIL fiber possessed comparable precisions (RSD) for all the tested PAHs and LODs for the high-ring PAHs, but relatively lower LODs for low-ring PAHs (Table 2).

\subsection{Sample analysis}

The prepared PIL fiber was applied to analyze three environmental water samples including river water, ground water from a polluted site, and municipal sewage effluent. All the PAHs were below the detection limits, except that $3.2 \mu \mathrm{gl} \mathrm{l}^{-1}$ acenaphthene was detected in the ground water, as well as 0.33 and $0.32 \mu \mathrm{gl}^{-1}$

Table 2

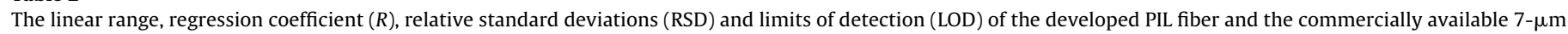
PDMS fiber in analysis of PAHs.

\begin{tabular}{|c|c|c|c|c|c|c|c|c|}
\hline \multirow[t]{2}{*}{ Sample name } & \multicolumn{4}{|c|}{ PDMS $(7 \mu \mathrm{m})$} & \multicolumn{4}{|l|}{ PIL } \\
\hline & $R$ & Linearity $\left(\mu g \mathrm{l}^{-1}\right)$ & RSD (\%) & $\operatorname{LOD}\left(\mu g \mathrm{l}^{-1}\right)$ & $R$ & Linearity $\left(\mu g \mathrm{l}^{-1}\right)$ & RSD (\%) & $\operatorname{LOD}\left(\mu \mathrm{gl}^{-1}\right)$ \\
\hline Nap & 0.993 & $0.5-20$ & 7.8 & 0.12 & 0.997 & $0.5-20$ & 12 & 0.10 \\
\hline Acpy & 0.990 & $0.5-20$ & 10 & 0.10 & 0.994 & $0.5-20$ & 13 & 0.06 \\
\hline Acp & 0.996 & $0.5-20$ & 9.8 & 0.10 & 0.997 & $0.5-20$ & 9.9 & 0.05 \\
\hline Flu & 0.997 & $0.5-20$ & 9.3 & 0.08 & 0.993 & $0.5-20$ & 9.2 & 0.05 \\
\hline Ant & 0.999 & $0.5-20$ & 8.8 & 0.11 & 0.998 & $0.5-20$ & 9.4 & 0.10 \\
\hline $\mathrm{Pa}$ & 0.999 & $0.5-20$ & 8.7 & 0.35 & 0.996 & $0.5-20$ & 13 & 0.25 \\
\hline $\mathrm{Fl}$ & 0.996 & $0.5-20$ & 9.4 & 0.10 & 0.998 & $0.5-20$ & 9.3 & 0.08 \\
\hline Pyr & 0.990 & $0.5-20$ & 9.7 & 0.10 & 0.998 & $0.5-20$ & 13 & 0.09 \\
\hline Baa & 0.998 & $0.5-20$ & 13 & 0.08 & 0.999 & $0.5-10$ & 12 & 0.10 \\
\hline Chr & 0.995 & $0.5-10$ & 10 & 0.06 & 0.999 & $0.5-10$ & 12 & 0.05 \\
\hline Bbf & 0.990 & $0.5-10$ & 25 & 0.08 & 0.997 & $0.5-10$ & 22 & 0.11 \\
\hline Bkf & 0.963 & $0.5-10$ & 16 & 0.10 & 0.966 & $0.5-10$ & 21 & 0.12 \\
\hline Bap & 0.992 & $0.5-10$ & 20 & 0.15 & 0.990 & $0.5-10$ & 18 & 0.18 \\
\hline Bjhip & 0.997 & $0.5-10$ & 26 & 0.11 & 0.986 & $0.5-10$ & 23 & 0.08 \\
\hline Dba & 0.988 & $0.5-10$ & 30 & 0.14 & 0.991 & $0.5-10$ & 29 & 0.10 \\
\hline Ind & 0.976 & $0.5-10$ & 26 & 0.09 & 0.973 & $0.5-10$ & 24 & 0.11 \\
\hline
\end{tabular}


Table 3

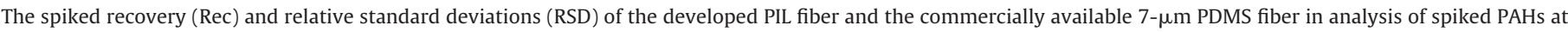
levels of 0.5 and $1.0 \mu \mathrm{g} \mathrm{l}^{-1}$.

\begin{tabular}{|c|c|c|c|c|c|c|c|c|}
\hline \multirow[t]{3}{*}{ Sample name } & \multicolumn{4}{|c|}{$0.5 \mu g \mathrm{l}^{-1}$} & \multicolumn{4}{|l|}{$1 \mu g \mathrm{l}^{-1}$} \\
\hline & \multicolumn{2}{|l|}{ PIL } & \multicolumn{2}{|c|}{ PDMS $(7 \mu \mathrm{m})$} & \multicolumn{2}{|l|}{ PIL } & \multicolumn{2}{|c|}{ PDMS (7 $\mu \mathrm{m})$} \\
\hline & $\operatorname{Rec}(\%)$ & RSD (\%) & $\operatorname{Rec}(\%)$ & RSD (\%) & $\operatorname{Rec}(\%)$ & RSD (\%) & $\operatorname{Rec}(\%)$ & RSD (\%) \\
\hline Nap & 54.1 & 10 & 69.6 & 7 & 105 & 9 & 80.3 & 7 \\
\hline Aсру & 100 & 4 & 92.5 & 5 & 103 & 79 & 91.2 & 5 \\
\hline Acp & 69.1 & 3 & 92.7 & 7 & 104 & 6 & 93.9 & 4 \\
\hline Flu & 92.0 & 2 & 86.7 & 7 & 96.2 & 6 & 90.0 & 4 \\
\hline Ant & 80.5 & 2 & 79.6 & 8 & 83.3 & 3 & 88.1 & 2 \\
\hline $\mathrm{Pa}$ & 103 & 1 & 95.0 & 7 & 86.4 & 4 & 97.4 & 2 \\
\hline $\mathrm{Fl}$ & 88.8 & 2 & 101 & 10 & 91.8 & 1 & 103 & 6 \\
\hline Pyr & 86.1 & 4 & 101 & 10 & 86.7 & 1 & 104 & 5 \\
\hline Baa & 94.0 & 5 & 85.2 & 18 & 89.1 & 7 & 113 & 5 \\
\hline $\mathrm{Chr}$ & 90.7 & 4 & 87.9 & 18 & 86.8 & 6 & 114 & 9 \\
\hline Bbf & 78.6 & 15 & 59.8 & 28 & 86.3 & 17 & 130 & 11 \\
\hline Bkf & 85.3 & 15 & 59.3 & 28 & 97.7 & 16 & 123 & 13 \\
\hline Bap & 73.3 & 2 & 57.8 & 35 & 81.0 & 2 & 130 & 5 \\
\hline Bjhip & 49.6 & 21 & 40.8 & 21 & 77.1 & 12 & 116 & 20 \\
\hline Dba & 50.5 & 20 & 46.0 & 22 & 87.1 & 2 & 128 & 20 \\
\hline Ind & 53.3 & 20 & 60.6 & 20 & 77.7 & 4 & 90.5 & 24 \\
\hline
\end{tabular}

naphthalene were detected in the effluent and the ground water, respectively.

For further evaluating the performance of the applicability of the proposed fiber in real sample analysis, the spiked recoveries of PAHs in these real water samples were determined by the PIL fiber and the commercial PDMS fiber, respectively. Table 3 shows the recoveries and precisions for the analysis of municipal sewage effluents at spiking levels of $0.5 \mu \mathrm{gl}^{-1}$ and $1 \mu \mathrm{gl}^{-1}$ each of the PAHs, respectively. As can be seen, this prepared PIL fiber had comparable performance with the commercial PDMS fiber. Similar results were also obtained for river water and ground water samples. For example, the respective recoveries of PAHs in river water at $0.5 \mu \mathrm{g} \mathrm{l}^{-1}$ spiked level were in the range of $52.5-111 \%$ for the PIL fiber and $57.4-103 \%$ for the PDMS fiber, while that in ground water at $5 \mu \mathrm{g} \mathrm{I}^{-1}$ spiked level were 53.3-105\% for the PIL fiber and 58.2-95.0\% for the PDMS fiber.

\section{Conclusions}

We demonstrated here for the first time the fabrication of covalent bond-based PIL SPME fibers with stainless steel fibers. By sequential coating the stainless steel fiber with gold film, $\mathrm{SiO}_{2}$ film, and 1-vinyl-3-(3-triethoxysilylpropyl)4,5-dihydroimidazdium chloride, a PIL film was successfully immobilized on the stainless steel fiber by covalent bond instead of physical adsorption. Due to the thermal stability of PILs were covalent bonded onto the mechanically strong stainless wire, this new SPME fiber possess excellent thermal stability and long lifetime. In addition, this developed PIL fiber exhibited comparable analytical performance to commercial PDMS fiber in the extraction of PAHs, which was attributed to the excellent extractability of PILs for PAHs. This developed procedure has great potential in fabrication of covalent bond-based SPME fibers.

\section{Acknowledgements}

This work was supported by the High-Tech Research and Development Program of China (2009AA061603), the National Natural Science Foundation of China (21025729, 20921063), and the China National Special Research Fund for Non-Profit Sector of Environmental Protection (201009015).

\section{Appendix A. Supplementary data}

Supplementary data associated with this article can be found, in the online version, at doi:10.1016/j.chroma.2012.01.052.

\section{References}

[1] K.R. Seddon, A. Stark, M.J. Torres, Pure Appl. Chem. 72 (2000) 2275.

[2] T. Welton, Chem. Rev. 99 (1999) 2071.

[3] J. Dupont, R.F. deSouza, P.A.Z. Suarez, Chem. Rev. 102 (2002) 3667.

[4] H. Ohno, K. Fukumoto, Electrochemistry 76 (2008) 16.

[5] V.I. Parvulescu, C. Hardacre, Chem. Rev. 107 (2007) 2615.

[6] C.F. Poole, S.K. Poole, J. Sep. Sci. 34 (2011) 8.

[7] P. Sun, D.W. Armstrong, Anal. Chim. Acta 661 (2010) 1.

[8] S. Pandey, Anal. Chim. Acta 556 (2006) 38.

[9] J.F. Liu, J.A. Jonsson, G.B. Jiang, Trends Anal. Chem. 24 (2005) 20

[10] J.F. Liu, G.B. Jiang, Y.G. Chi, Y.Q. Cai, Q.X. Zhou, J.T. Hu, Anal. Chem. 75 (2003) 5870.

[11] X. Han, D.W. Armstrong, Acc. Chem. Res. 40 (2007) 1079

[12] C.F. Poole, S.K. Poole, J. Chromatogr. A 1217 (2010) 2268.

[13] E. Aguilera-Herrador, R. Lucena, S. Cardenas, M. Valcarcel, Trends Anal. Chem. $29(2010) 602$

[14] D.W. Armstrong, L. He, Y.S. Liu, Anal. Chem. 71 (1999) 3873.

[15] E.G. Yanes, S.R. Gratz, M.J. Baldwin, A.M. Stalcup, Anal. Chem. 73 (2001) 3838.

[16] L.J. He, W.Z. Zhang, L. Zhao, X. Liu, S.X. Jiang, J. Chromatogr. A 1007 (2003) 39.

[17] D.W. Armstrong, L.K. Zhang, L.F. He, M.L. Gross, Anal. Chem. 73 (2001) 3679.

[18] S. Dai, Y.H. Ju, C.E. Barnes, J. Chem. Soc., Dalton Trans. (1999) 1201.

[19] A.E. Visser, R.P. Swatloski, W.M. Reichert, S.T. Griffin, R.D. Rogers, Ind. Eng. Chem. Res. 39 (2000) 3596.

[20] L. Xu, C. Basheer, H.K. Lee, J. Chromatogr. A 1152 (2007) 184.

[21] M.A. Jeannot, A. Przyjazny, J.M. Kokosa, J. Chromatogr. A 1217 (2010) 2326.

[22] J.F. Liu, Y.G. Chi, G.B. Jiang, T. Chao, J.F. Peng, J.T. Hu, J. Chromatogr. A 1026 (2004) 143.

[23] J.F. Liu, N. Li, G.B. Jiang, J.M. Li, J.A. Jonsson, M.J. Wen, J. Chromatogr. A 1066 (2005) 27.

[24] Y.N. Hsieh, P.C. Huang, I.W. Sun, T.J. Whang, C.Y. Hsu, H.H. Huang, C.H. Kuei, Anal. Chim. Acta 557 (2006) 321.

[25] F. Zhao, Y.J. Meng, J.L. Anderson, J. Chromatogr. A 1208 (2008) 1.

[26] Q.C. Zhao, J.C. Wajert, J.L. Anderson, Anal. Chem. 82 (2010) 707

[27] T.D. Ho, A.J. Canestraro, J.L. Anderson, Anal. Chim. Acta 695 (2011) 18.

[28] S. Lee, Chem. Commun. (2006) 1049.

[29] C.P. Mehnert, R.A. Cook, N.C. Dispenziere, M. Afeworki, J. Am. Chem. Soc. 124 (2002) 12392.

[30] M.H. Valkenberg, C. deCastro, W.F. Holderich, Green Chem. 4 (2002) 88.

[31] M. Gruttadauria, S. Riela, L.P. Meo, F. Danna, R. Noto, Tetrahedron Lett. 45 (2004) 6113.

[32] Y.S. Chi, J.K. Lee, S. Lee, I.S. Choi, Langmuir 20 (2004) 3024.

[33] X.D. Mu, J.Q. Meng, Z.C. Li, Y. Kou, J. Am. Chem. Soc. 127 (2005) 9694.

[34] L.M. Liz-Marzan, M. Giersig, P. Mulvaney, Langmuir 12 (1996) 4329.

[35] R. Marcilla, J.A. Blazquez, J. Rodriguez, J.A. Pomposo, D. Mecerreyes, J. Polym Sci., Part A: Polym. Chem. 42 (2004) 208.

[36] G.Q. Lai, J.J. Peng, J.Y. Li, H.Y. Qiu, J.X. Jiang, K.Z. Jiang, Y.J. Shen, Tetrahedron Lett 47 (2006) 6951. 